\title{
Generality and Specificity of the Binding Behaviour of Lysozyme with Pectin Varying in Local Charge Density and Overall Charge
}

\author{
Yurij A. Antonov*, Irina L.Zhuravleva \\ N.M. Emanuel Institute of Biochemical Physics, Russian Academy of Sciences, Kosygin Str. 4. 119334 \\ Moscow, Russia \\ Miete Celus, Clare Kyomugasho \\ Centre for Food and Microbial Technology, Faculty of Bioscience Engineering, KU Leuven, Kasteelpark \\ Arenberg 22, Box 2457, B3001 Leuven, Belgium \\ Salvatore Lombardo, Wim Thielemans
}

Renewable Materials and Nanotechnology Group, University of Leuven Kulak Kortrijk Campus, Etienne Sabbelaan 53, Box 7659, 8500 Kortrijk, Belgium

Marc Hendrickx

Centre for Food and Microbial Technology, Faculty of Bioscience Engineering, KU Leuven, Kasteelpark Arenberg 22, Box 2457, B3001 Leuven, Belgium

Paula Moldenaers

Soft Matter Rheology and Technology, Department of Chemical Engineering, KU Leuven, Celestijnenlaan 200f, Box 2424, B3001 Leuven, Belgium

Ruth Cardinaels

Polymer Technology, Department of Mechanical Engineering, TU Eindhoven, Box 513, 5600 MB Eindhoven, The Netherlands

*Corresponding author: Yurij A. Antonov, e-mail:chehonter@yandex.ru

Abstract We examined the similarities and differences of the binding behavior of lysozyme (lys) with pectin varying in local charge density (blockwise distribution and statistical distribution of methoxyl groups, BP and SP) and similar degree of methoxylation (DM). The interaction at ionic strength $I=0.01$ and $\mathrm{pH} 5.1$ was found to be mainly electrostatic, associated with an exothermic enthalpy change. BP which is more inclined to self-association binds lys forming strongly associated complex particles with average sizes much larger $(10-20 \mu \mathrm{m})$ than that of SP. The latter formed with lys small $(0.6-3 \mu \mathrm{m})$ complex particles or liquid droplets depending on DM. The critical Iset and pHset values for BP/lys systems, above which complexes do not form, are not sensitive to DM (approximately 0.1 and 11.5 respectively), whereas for SP/lys systems the critical Iset and pHset values decrease significantly with increasing DM. The effect of I on complexation (CP) of lys with BP and SP has a nonmonotonic character. The distribution of BP and SP within the complex particles is irregular and is different for pectin with low and high DM values. The binding constant and stoichiometry ratio (lys/pectin) of the systems with SP are slightly higher 
than those of BP at the same DM, and these values decrease sharply (50 times) with increasing DM. The large positive changes in entropy in SP/lys systems suggest that the interactions in these systems tend to be more hydrophobic in character at high DM. On the other hand, with BP, a negative enthalpy change, especially for pectin with low charge, suggests the formation of a large quantity of hydrogen bonds or van der Waals interactions during CP.

Keywords: Pectin; lysozyme; complexation; confocal microscopy; ITC; structure.

\section{INTRODUCTION}

Proteins, polysaccharides and some synthetic and modified polymers are widely used in the food, pharmaceutical, and cosmetic industries in the form of multicomponent systems (Stephen, Phillips, Williams, 2006). At present, it is generally accepted that intermolecular interactions and the phase state of these systems determine their functional properties (Tolstoguzov, 1986). Phase separation in aqueous protein-polyelectrolyte (PE) systems can be the result of the manifestation of two different physicochemical phenomena: thermodynamic incompatibility of the (bio)polymers and formation of water insoluble protein-polyelectrolyte complexes. These phenomena are frequently described as segregative and associative phase separation (Piculell, Lindman, 1992; De Kruif, C. G., \& Tuinier, R. 2001). There is a large number of original studies and reviews concerning interaction and complex formation in protein-synthetic PE systems (Tsuboi, et al, 1996; Xia, Dubin, Dautzenberg, 1993; Mattison, Brittain, Dubin, 1995; Cooper, Dubin, Kayitmazer, \& Turksen,. 2005; Da Silva, Lund, Jönsson \& \& Akesson, 2006; Kaibara, Okazaki, Bohidar, \& Dubin, 2000; Mattison, Dubin, \& Brittain, 1998; Seyrek, Dubin, Tribet, \& Gamble, 2003; Kayitmazer et al., 2013; Cooper, Dubin, Kayitmazer, \& Turksen, 2005) and protein-polysaccharide systems (Tolstoguzov, 1997,1986, 2003;Antonov, Zhuravleva Cardinaels, Moldenaers, 2015; Antonov, Cardinaels, Moldenaers, 2017; Antonov, Zhuravleva, Cardinaels, Moldenaers. 2018; Galazka, Smith, Ledward, \& Dickinson,1999; Tolstoguzov, 1986; Serov, Antonov, Tolstoguzov, 1985; Gurov, Gurova, Leontiev, Tolstoguzov, 1988; Laneuville, Paquin, Turgeon, 2000; Dickinson, \& Pawlowsky, ,1998; Weinbreck, De Vries, Schrooyen, \& De Kruif, 2003; Doublier, Garnier, Renard, \& Sanchez, 2000; Kabanov, 1994). The nature of protein/polysaccharide complexes is influenced by entropic factors, that depend on parameters such as the structure and the molecular weight of the biopolymers. The complexes are also influenced by enthalpic forces, which are regulated by the protein/polysaccharide ratio and the nature and density of charges as well as the type and distribution of functional groups on the biopolymers (Girard, Turgeon and Gauthier 2003). 
Effects of experimental parameters such as $\mathrm{pH}$ and ionic strength, as well as that of many structural parameters (polyion and polyampholyte types, their flexibility) on complexation in protein/PE systems have been extensively studied for many types of proteins. On the contrary, the effects of the local structural changes of the PE (the number of charged and uncharged groups, and their distribution along the chain) have only been investigated for a limited amount of proteins. In this connection we can refer to studies on the complexation between pectin and $\beta$-lactoglobulin (Sperber, Schols, Stuart, Norde, Voragen, 2009; Girard, Turgeon, \& Gauthier 2003a, b; Xu, Melton, Jameson, Williams, Mc Gillivray 2015; Xu, Melton, Ryan, 2018). In principle local structural changes can significantly alter the charge density of the PE and its chain stiffness and can thus have a great impact on protein/PE complexation (CP). Lys (E.C.3.2.17, N-acetylmuramic-hydrolase) is a bacteriolytic enzyme commonly found in nature in many plant and animal sources, which is especially abundant in hen egg whites. It is a compact basic (pI=11.32) globular protein having an ellipsoidal shape with dimensions of $4.5 \times 3.0 \times 3.0 \mathrm{~nm}$ and a molecular weight $\mathrm{Mw}=14.3 \mathrm{kDa}$. Lys is found mainly as a monomer, but like many other natural compounds, it might even be more active in the dimeric or polymeric form. Bacteriostatic and bactericidal properties of lys are used to preserve various types of food (Cegielska-Radziejewska, Leśnierowski, Kijowski, 2008; Proctor, and Cunningham, 1988). Pectin at first approximation is a linear polysaccharide comprising galacturonic acid residues. It is mainly found in the middle lamella of the plant cell membrane. Some of its galacturonate units are esterified as methyl galacturonate, whose extent is reported as the degree of methoxylation (DM). These methylesters can be present with a statistical or blockwise distribution (Kohn, and Luknar, 1977; Kohn, Markovič, Machová, 1983; Kohn, 1987; Williams, Buffet, Foster, Norton, 2001; Daas Meyer Hansen, Schols, De Ruiter, Voragen, 1999; Ngouémazong, Tengweh, Duvetter, Fraeye, Van Loey, Moldenaers, Hendrickx 2011). It is well-known that pectin with a blockwise distribution of methylesters is more inclined to self-association than pectin with a statistical distribution of methylesters (Antonov et al., 1996). Its excellent functional properties make pectin a widely used additive in the food industry e.g. as jam, jelly, emulsion stabilizer and thickener in sauces.

In our recent study we characterized the CP of pectin with lysozyme (lys) at different physicochemical conditions and compositions of the system (Antonov et al, 2019). The effect of the overall charge of pectin (DM) on the complexation behavior was investigated. The work presented in the present paper focusses on the binding behavior of lys with pectin varying in local charge density (block wise distribution and statistical distribution of methoxyl groups denoted as BP and SP) but being similar in overall charge. Analysis of the lys protein surface reveals a close to homogeneous distribution of positive charges over the surface (Van de Weert, Andersen and Frokjaer, 2004), suggesting that the interaction with pectin does not occur at a very specific site on the lysozyme 
molecule. This fact combined with the potential applications of pectin/lysozyme systems provides a strong motivation for this study.

Turbidimetric titration, dynamic light scattering (DLS), confocal and optical microscopy, and isothermal titration calorimetry (ITC) were applied. Turbidimetric titration and DLS allowed to determine the sizes of the complex particles, and the main phase transitions of the system in terms of composition, $\mathrm{pH}$ and ionic strength. Confocal and optical microscopy allowed to monitor changes of the system morphology and to determine the distribution of the biopolymers within the complex particles. ITC allowed accurate measurements of the thermodynamic parameters, such as the binding constant, enthalpy, entropy, and stoichiometry of the interactions.

\section{MATERIALS AND METHODS}

\subsection{Materials}

\subsubsection{Biopolymers and reagents}

The lys from chicken egg white (dialyzed, lyophilized powder) was purchased from SigmaAldrich and used without further purification. High methylesterified commercial citrus pectin with molar mass of $56.1 \mathrm{kDa}$ and DM of 95.1 was obtained from Sigma-Aldrich (Belgium). Pectins with a blockwise distribution of the methyl ester groups (BP) and various degrees of methoxylation (DM) namely 16.9\% (BP16.9), 33.1\% (BP33.1) and 68.2\% (BP68.2) were produced enzymatically using plant pectin methyl esterase (PME). The enzymatic demethylesterification included an incubation of highly methyl esterified citrus pectin (DM of 95.1\%) with purified carrot PME at 30 ${ }^{\circ} \mathrm{C}$ for predetermined time periods. Pectins with a statistical distribution of methyl ester groups (SP) and various DM values namely 16.2\% (SP16.2), 38.2\% (SP38.2), 66.5\% (SP66.5) and 77.7\% (SP77.7) were produced chemically from highly methylesterified citrus pectin (DM of 95.1\%) by titration with predetermined amounts of $0.1 \mathrm{M} \mathrm{NaOH}$ solution at $4{ }^{\circ} \mathrm{C}$ and a pH of 11 . The detailed procedure for the preparation of BP and SP samples can be found in earlier work (Celus et al. 2017). After preparation, the resulting solutions were adjusted to $\mathrm{pH} 6$, dialyzed (Spectra/Por®, MWCO $=12-14 \mathrm{kDa}$ ) for $48 \mathrm{~h}$ against demineralized water and subsequently lyophilized. Pectin from each batch was characterized in terms of DM using Fourier transform infra-red spectroscopy (FT-IR) (Shimadzu FTIR-8400S, Japan), as in Celus et al. (2017). The molar mass distribution profile of all samples did not reveal any depolymerisation as no shift in elution time was observed on HPSEC chromatograms. The average molar mass of the samples, as determined by HPSEC chromatography was $44.6 \pm 3.5 \mathrm{kDa}$ and varied between $41.1 \mathrm{kDa}$ and $48.1 \mathrm{kDa}$ whereas the mother pectin had a molar mass of $56.1 \mathrm{kDa}$ (Celus et al., 2017). All reagents were of analytical reagent grade. Milli-Q ultrapure water was used in all experiments. 

mixtures. To prepare solutions of lys and pectin with the required concentrations, the weighed amount of biopolymer sample was gradually added to an acetic acid-sodium acetate buffer ( $\mathrm{pH}$ 5.1 , ionic strength $I=0.01$ ) at $23{ }^{\circ} \mathrm{C}$ and stirred for $1 \mathrm{~h}$. The resulting solutions were centrifuged at $50000 \mathrm{~g}$ and $23{ }^{\circ} \mathrm{C}$ for $1 \mathrm{~h}$ to remove insoluble particles. The lys content in the stock solution was determined by means of UV absorption using the extinction coefficient for highly purified lys which is $2.64 \mathrm{ml} \mathrm{mg}^{-1} \mathrm{~cm}^{-1}$ at $281.5 \mathrm{~nm}$ (Aune, \& Tanford, 1969). To study the effect of $\mathrm{pH}$ on the complexation behavior, the $\mathrm{pH}$ of the complex mixtures was changed in a range from 5.0 to 11.0 by addition of $0.1-0.5 \mathrm{M} \mathrm{NaOH}$. To study the effect of $I$ on the complexation behavior, the $I$ of the complex mixtures was changed in a range from 0.01 to 0.5 by addition of $3.5 \mathrm{M} \mathrm{NaCl}$. Concentrations of pectin in the stock solutions were determined by drying at $104{ }^{\circ} \mathrm{C}$ to constant weight. Solutions of the biopolymers were kept at least overnight in the fridge to allow for full hydration of the molecules. To prepare mixed solutions of lys with pectin with the required concentrations, weighed amounts of the pectin stock solution were added to the lys stock solution at $23{ }^{\circ} \mathrm{C}$.

\subsection{Methods}

\subsubsection{Turbidity measurements}

Turbidity values of the systems as a function of the pectin/lys weight ratio (q) were measured at $500 \mathrm{~nm}$ using a UNICCO SQ2800 UV/VIS spectrometer. The error of the turbidity measurements was typically about $2 \%-3 \%$. In the weight ratio range from 0.1 to 0.6 the errors were markedly larger (6-8\%). With increasing $\mathrm{q}$, the $\mathrm{CP}$ behavior can be characterized by three transitions identified as $\mathrm{q}_{\text {onset, }} \mathrm{q}_{\phi}$, and $\mathrm{q}_{\max }$ (Carlsson, et al., 2001). These correspond to respectively the transition from the absence of $\mathrm{CP}$ to the formation of water soluble complexes, from water soluble complexes to water insoluble complexes and their phase separation, and maximal CP. To obtain accurate values for these transition points, additional characterizations were performed, as described in detail in our recent work (Antonov et al. 2019).

\subsubsection{Light Scattering}

Determination of the intensity size distribution functions of pectin and lys solutions as well as pectin/lys mixtures was performed by means of dynamic light scattering (DLS) with an ALV/CGS-3 compact goniometer system (ALV GmbH, Germany). The measurement procedure has been described in detail in our earlier work (Antonov et al, 2017). From these measurements, the scattering intensity averaged hydrodynamic radius was determined, assuming a spherical shape of the complexes.

\subsubsection{Bright light microscopy.}


Microscopy observations during CP have been performed, using an Olympus BX51W1 fixed stage microscope equipped with a high resolution CCD-camera, (1000x1000 pixels, C-8800-21, Hamamatsu), using different magnifications.

\subsubsection{Fluorescent imaging}

Fluorescent imaging was performed using a multi-beam confocal microscope (VisiTech, UK), equipped with an oil-immersion objective (20x, 0.85 NA, Olympus, Japan) using $532 \mathrm{~nm}$ and $642 \mathrm{~nm}$ as excitation wavelengths. Before imaging, pectin and lys were fluorescently labeled by storing a pectin solution containing Atto $647 \mathrm{~N}$ dye (ATTO Tec. Germany) at $5^{\circ} \mathrm{C}$ during 3 days, whereas a lys solution containing Rhodamine B dye was kept under the same conditions. This labeling allowed to spectrally separate the signal from lys (red) and pectin (green). Image analysis was performed using Image J v1.43r software. More than 100 images were analyzed and representative ones are presented here. Using turbidity characterization it was verified that the fluorescent labelling has no significant effect on the complexation process.

\subsubsection{Isothermal Titration Calorimetry (ITC)}

Calorimetric experiments were performed using a TAM III isothermal calorimeter (TA Instruments). All solutions used for ITC were dialyzed against the buffer during $48 \mathrm{~h}$ at $4^{\circ} \mathrm{C}$. The samples were degassed by sonicating the solutions for 15 minutes under vacuum. The system was equilibrated at $25^{\circ} \mathrm{C}$ while stirring at $120 \mathrm{rpm}$ until the baseline shift was $<50 \mathrm{nW} / \mathrm{h}$. The pectin solution in the reaction cell $(0.8 \mathrm{~mL})$ was titrated with 13 successive $15-\mu \mathrm{L}$ injections of lys solution. Each addition lasted $10 \mathrm{~s}$ with an interval of $20 \mathrm{~min}$ between consecutive injections. This long resting time between injections was necessary to reach thermodynamic equilibrium. This equilibrium was attained when no more energy was released or absorbed in the reaction cell. The heat of dilution from the blank titration of the lys into the buffer solution was subtracted from the raw data. Measurements were carried out two to five times, depending on the sample. The thermodynamic parameters, including the binding constant $(K)$, enthalpy $(\Delta H)$, entropy $(\Delta S)$, and binding stoichiometry $(n)$ (molar ratio monomeric lys/pectin), were calculated by curvefitting of the binding isotherms with a Levenberg-Marquardt nonlinear regression model. Thereto, an independent binding site interaction model with a single binding site was used (Freyer \& Lewis, 2008, Brown 2009). This model includes a mass balance:

$L_{t}=[L]+n \Theta M_{t}$

where $L_{t}$ is the starting concentration of ligand $\mathrm{L},[L]$ is the free ligand concentration, $M_{t}$ is the total macromolecule concentration, $\mathrm{n}$ is the binding stoichiometry and $\Theta$ is the fraction of sites occupied by the ligand, whereby the amount of free and bound ligand depends on the association equilibrium constant $\mathrm{K}$ : 
$209 \quad \mathrm{~K}=\frac{\Theta}{(1-\Theta)[L]}$

210 Combining equations 1 and 2 gives:

$211 \Theta^{2}-\Theta\left[1+\frac{L_{t}}{n M_{t}}+\frac{1}{n K M_{t}}\right]+\frac{L_{t}}{n M_{t}}=0$

212 The total heat produced $Q$ is described by Equation (4), with $\mathrm{V}_{0}$ the initial volume of the sample 213 cell:

$214 Q=n \Theta \Delta H M_{t} V_{0}$

215 Solving Equation (3) for $\Theta$ and then substituting this into Equation (4) gives:

216

$Q=\frac{n M_{t} \Delta H V_{0}}{2}\left[1+\frac{L_{t}}{n M_{t}}+\frac{1}{n K M_{t}}-\sqrt{\left(1+\frac{L_{t}}{n M_{t}}+\frac{1}{n K M_{t}}\right)^{2}-\frac{4 L_{t}}{n M_{t}}}\right]$

217 Equation (5) only applies to the liquid contained in volume $\mathrm{V}_{\mathrm{o}}$. Therefore, after completing an 218 injection, a correction must be made for the increase in volume. Equation (6) then accounts for the 219 heat released, $\Delta \mathrm{Q}(\mathrm{i})$, from the $\mathrm{i}^{\text {th }}$ injection, taking into account the increase in volume due to the 220 injected volume:

$221 \Delta \mathrm{Q}(\mathrm{i})=Q(i)+\frac{d V_{i}}{V_{0}}\left[\frac{Q(i)+Q(i-1)}{2}\right]-Q(i-1)$

222 The NanoAnalyze data analysis software (TA instruments) was used to perform the data analysis.

223 Based on these data, the Gibbs free energy is calculated from the binding constant:

224

$\Delta G=-R T \ln K$

225

and subsequently the entropy change can be found from:

226

$\Delta G=\Delta H-T \Delta S$

\subsubsection{Statistical analysis}

DLS measurements of the intensity size distribution functions were carried out in duplicate and results are averages of five independent runs. Each turbidimety curve was obtained by taking the average of four measurements. Calorimetric measurements were carried out two to five times, depending on the sample. The obtained results were analysed using the Origin 9 software (Microcal, USA).

\section{Results and discussion}

\subsection{Determination of the main CP parameters ( $q_{\text {onset }}, q_{\phi,} q^{*}{ }_{\phi}$, and $\left.q_{\max }\right)$ via turbidimetry}

First, turbidimetry was applied to determine the main CP parameters of mixtures containing lys and $\mathrm{BP}$ varying in DM. A series of more than 50 mixtures comprising lys and $\mathrm{BP}$ with various pectin/lys weight ratios (q) were prepared at $\mathrm{pH} 5.1$ and ionic strength $I=0.01$, and stirred for 120 min to reach the equilibrium state. The turbidimetry data of these systems at $500 \mathrm{~nm}\left(\tau_{500}\right)$ as a function of $\mathrm{q}$ are presented in Figures 1 and 2. The thin dash dot lines in Figures 1 show the data 
241 for the SP/lys system from our previous study (Antonov et al. 2019) for comparison. At first glance

242 a comparison between the data obtained for BP16.9/lys, BP33.1/lys and BP68.2/lys systems and 243 that for SP16.2/lys, SP38.2/lys and SP66.5/lys systems studied recently shows that replacing pectin with a statistical distribution of methyl ester groups with pectins with a blockwise distribution of methylester groups at the same DM values does not appreciably affect the turbidimetry results (Fig. 1).

\section{Figure 1}

An addition of even a small amount of a 0.04 wt\% BP16.9 or BP33.1 solution in a 0.04 wt\% lys solution results in the appearance of turbidity whereby the q values exceed the qonset value. As usual, the turbidity measurements reveal five domains of q (Fig 1a', a", Fig 1b', b", Fig 2a', a") corresponding to (I) the absence of CP, (II) soluble complex formation, (III) phase separation of an insoluble complex (Carlsson et al, 2001), (IV) suppression of phase separation, and (V) the absence of CP. The transition from region I to region II is denoted "qonset", the transition from region II to region III is denoted " $\mathrm{q}_{\phi}$ ", the transition from region III to region IV is denoted " $\mathrm{q}^{*}$ ", and the transition from region IV to region $\mathrm{V}$ is denoted " $\mathrm{q}_{\text {set }}$ ". The absence of $\mathrm{CP}$ at $\mathrm{q}>\mathrm{q}_{\text {set }}$ does not mean complete absence of interactions between the ion pairs of the protein and polysaccharide. Electrostatic complexation of proteins with polysaccharides includes at least two stages, first the formation of salt bonds between the amino groups of the protein and negatively charged (in the case of pectin) groups of the polysaccharide, and subsequently the aggregation of the complexes by secondary forces due to the decrease of the zeta potential of the system. At $q>q_{\text {Set }}$ association and aggregation of the primary complex pairs is impossible due to the highly negative charge of the complex system.

The $\mathrm{q}_{\phi}$ and $\mathrm{q}^{*} \phi$ values are a measure of the complex stability and characterize the phase boundary. The $\mathrm{q}_{\text {onset }}$ and $\mathrm{q}_{\phi}$ values as a function of DM for BP/lys systems are presented in Figure 2. Similar to SP (Antonov et al, 2019) BP interacts with lys starting from an extremely low weight ratio of pectin in the mixtures namely $\mathrm{q}_{\text {onset }}=7 \cdot 10^{-4}$ for highly charged BP16.9 and ranges to $3 \cdot 10^{-3}$ for BP68. Water soluble complexes are formed in a narrow q range between $7 \cdot 10^{-4}$ and $1.410^{-2}$ depending on the DM values, as shown in Figure 2. 
The turbidimetry data clearly show that differences between systems with BP and SP exist only

273 for mixtures with lowly charged polysaccharides (high DM). The main CP parameters including qMax obtained for BP16.9/lys versus SP16.2/lys and BP33.1/lys versus SP38.2/lys are the same at corresponding DM values, as show in Figure 1. However the qMax value for the BP68.2/lys system, which characterizes at first approximation the stoichiometric composition of the complexes, is appreciably higher (0.37) than that of the SP66.5/lys system. In other words, the stoichiometric composition for the former system is observed at a larger content of pectin (Fig. 1c'"). Taking into account that the weight average molecular weight of Lys is $14.3 \mathrm{kDa}$ (Rezwan, Meier, Gauckler, 2005 ) and that of pectin is $44.6 \pm 3.5 \mathrm{kDa}$, we can roughly evaluate the "molar" ratio pectin/lys in the complex phases. A simple calculation shows that the lys/pectin ratio is approximately $8: 1$ mole lys/mole pectin for the BP68.2/lys system, which is less than that of the SP66.5/lys system (10:1) at almost the same DM value of functional groups. Moreover, for these lowly charged systems, liquid complex coacervates were observed for the SP/lys mixtures whereas the systems with BP resulted in water insoluble complex particles for all studied conditions.

\subsection{Size of complex particles}

DLS was applied to characterize the scattering intensity average size of the complex particles at different DM values. All measurements were performed at a constant total concentration of biopolymers $(0.04 \mathrm{wt} \%)$ in a q range from 0.05 to 1.0 . The scattering intensity size distribution functions of the BP/lys systems are presented in Figures 3 a-c. For comparison, the thin line shows the scattering intensity size distribution function of a SP/lys system at the corresponding DM values and for $\mathrm{q}=\mathrm{q}_{\text {Max. }}$. The scattering intensity averaged hydrodynamic radius of all pectin particles was approximately 104-114 nm depending on the DM of pectin (Fig. 3a-c). Hence, the effect of the methylester distribution on the size of the pure pectin is negligible under the studied conditions. The average radius of the lys monomer was $1.9 \mathrm{~nm}$ (data not shown), in accordance with other known data (Parmar, \& Muschol, 2009; Valstar, Brown, Almgren, 1999). Since the average size of pectin is much larger than that of lys, changes in the sizes of the complex particles can easily be monitored. For all studied systems the presence of even a small amount of pectin in the lys solution (at $\mathrm{q}=0.05$ ), leads to a significant shift of the dominant peak to a larger average particle radius The BP/lys particles are significantly larger than those of the SP/lys systems at the same DM value (Figure 3d). Hence, based on the DLS results one important difference in the CP of lys with SP and BP can be pinpointed. The complexes obtained with BP are more aggregated as compared to those obtained with SP at the same DM. This can be due to the higher capacity of BP to aggregate in the acidic $\mathrm{pH}$ range in the presence of monovalent ions which induce aggregation of stiffened BP chains. Such aggregation can arise from e.g. cooperative 
attraction, or via entanglement of rigid structures (Ström, Schuster, MengGoh, 2014). The interactions between the blocks of the BP samples are more cooperative than those of SP. The solubility of the BP samples in our study was always less than the solubility of SP samples at the same DM values. However, for the pure pectins no significant size differences could be detected with DLS.

The dependence of the average sizes on q has an extremal character with the q value corresponding to the maximum particle size equal to or close to that determined by turbidimetry. At $q$ values above $q_{\text {Max }}(q=0.5-1.0)$ the sizes of the complex particles gradually become similar to that of free pectin molecules. The evolution of the size of the complexes with q together with the evolution of turbidity with q relate to the degree of binding (yield) of lys in the water insoluble complex phase with pectin. At $\mathrm{q}<\mathrm{q}_{\mathrm{Max}}$ the yield of lys in the complexes increases with increasing $\mathrm{q}$. At $\mathrm{q}>\mathrm{q}_{\mathrm{Max}}$ the yield of lys in the complex decreases with increasing q. At high q values, the secondary aggregation of complexes is hampered due to the highly charged nature of the formed complexes.

\subsection{Morphology of complex BP/lys systems}

Brightfield light microscopy images of complex BP68.2/lys particles at different q values and a total biopolymer concentration in the mixture of $0.04 \mathrm{wt} \%$ are presented in Figure 4 . The microscopy images clearly demonstrate the increase of the average size of the complex particles when the content of pectin increased up to qMax (Figure 4a-c). Separation of a complex BP68.2/lys system by centrifugation showed that the complex phase is a jelly-like soft material. In contrast to the BP/lys system the complex phases of the SP66.5/lys and SP77.7/lys systems are liquid and have a droplet morphology (Antonov et al, 2019). Hence, the microscopy images demonstrate that another main difference between the complex particles of the highly methoxylated BP/lys and SP/lys systems is the aggregation state (liquid or solid) of the complex phase.

\section{Figure 4}

\subsection{Structure of the complex particles}


Confocal Laser Scanning Microscopy (CLSM) was applied to monitor the distribution of biopolymers within the BP/lys complexes at different q values and the images are compared with those for SP/lys systems (Antonov et al, 2019) at the corresponding q values. Representative images are presented in Figures 5 and 6. For the BP16.9/lys system six characteristic compositions of the complex system were considered (Fig 5a-f) namely $q=0.05$, corresponding to an excess of lys, $\mathrm{q}=\mathrm{q}_{\max }$, corresponding to maximal complexation according to the turbidity analysis (Figs. 1), $\mathrm{q}=0.5$ and 0.9 , and two compositions characterized by an excess of pectin in the complex system ( $\mathrm{q}=1.5$ and 2.0). At $\mathrm{q} \leq \mathrm{q}_{\max }$ the intensity signal from the positively charged labelled lys protein (red) is dominating (Fig. $5 \mathrm{a}, \mathrm{b}$ ) whereas at $\mathrm{q}>\mathrm{q}_{\max }$ the intensity contribution from the labelled pectin (green) becomes dominant in the complex particles. Besides, the yellow signal corresponding to mutual compensation of both macro counterions is also present in most images. The results obtained for the BP16.9/lys systems show that the higher the content of pectin in the complex mixture the more the relative content of pectin within the complex particles. The structure of the BP33.1/lys system (data not presented) is quite similar to that of the BP16.9/lys system and SP16.2/lys system (Antonov et al, 2019). Since the composition of the complex particles for the BP16.9/lys and SP16.2/lys systems depends on the composition of the initial mixture, this implies that the reactivity of free pectin chains may be considered higher than that of pectin partially covered by lys (Antonov et al, 2019). The distribution of pectin and lys within the complex particles of the BP68.1/lys system differs from that of the BP16.9/lys as well as that from the SP66.5/lys system (Antonov et al, 2019). An increase of the BP68.1 content in the complex mixture (increase in q value) did not lead to an appreciable increase in the BP68.1 content within the complex particles (Fig. 6d). The later ones remain enriched in lys even at high q values. This indicates a difference in the mechanism of binding of lys with low methoxylated and highly methoxylated pectins similar to what we observed for SP/lys systems (Antonov et al, 2019). It is interesting to note that in the given system regions with complete compensation of the macro ions are very small (yellow color) even at $\mathrm{q}_{\max }$ corresponding to maximum complexation according to turbidimetry (Fig. 1). A comparison of the images for the BP68.1/lys (Fig. 6d) and the SP66.5/lys system (Antonov et al, 2019) clearly shows that the degree of irregularity in the distribution of the biopolymers within the complex particles is much higher for the former system. This can be a result of the additional aggregation of lys during $\mathrm{CP}$ with BP. This stronger aggregation of these complexes may also be the reason for the absence of liquid coacervation in BP/lys systems at high DM values which was observed for SP66.5/lys and SP77.7/lys systems (Antonov et al, 2019). 
372

373

374

375

376

377

378

379

380

381

382

383

384

385

386

387

388

389

390

391

392

393

394

395

396

397

398

399

400

401

402

\subsection{Effect of ionic strength and $p H$}

Figure $7 \mathrm{a}$ presents $\tau_{500}$ values of complex pectin/lys systems as a function of the $\mathrm{pH}$ obtained for BP with various DM values. All plots were determined at $\mathrm{q}$ values corresponding to $\mathrm{q}_{\mathrm{Max}}$ for each system at $I=0.01$. The results obtained and their comparison with those determined for SP/lys systems at corresponding conditions (Antonov et al, 2019) show at least one principal difference in their behavior. The minimal $\mathrm{pH}$ value at which $\tau_{500}$ becomes zero $\left(\mathrm{pH}_{\text {set }}\right)$, indicating the absence of CP, is not dependent on the DM value in BP/lys systems, i.e on the overall charge of the pectin molecules, whereas in SP/lys systems the $\mathrm{pH}_{\text {set }}$ value decreases with increasing DM.

These facts allow to state that the main factor determining CP in BP/lys systems in the alkaline $\mathrm{pH}$ range is the aggregation of pectin and the formed complex particles, steered by secondary forces, whereas in SP/lys systems the overall charge of pectin is the driving force determining CP. In the $\mathrm{pH}$ range from 5 to 9.5 the positive charge of lys decreases monotonically (Bharti, 2014) and the carboxyl groups of pectin are completely ionized (Kohn, 1973). Therefore decreasing $\tau_{500}$ values with increasing $\mathrm{pH}$ values from 5 to 9.5 were expected. However, strong aggregation of the complex particles under the given conditions extends the stability of the complexes to higher $\mathrm{pH}$ values. The origin of the forces responsible for the strong aggregation in BP/lys systems, especially in the alkaline range is not quite clear.

Figure 7

The effect of $I$ on the $\tau_{500}$ values of the BP/lys systems at different DM values is demonstrated in Figure 7b. One common feature of systems containing either BP or SP (Antonov et al, 2019) is that the minimal $I$ value at which $\tau_{500}$ becomes zero $\left(I_{\text {set }}\right)$, indicating the absence of $\mathrm{CP}$, is the same $\left(I_{\mathrm{set}}=0.1\right)$ for the highly charged $(\mathrm{DM}=16 \%) \mathrm{SP}$ and BP. For the highly charged BP16.9 and BP33.1 the dependence of $\tau_{500}$ on $I$ has a nonmonotonic character displaying a maximum in CP at $I \approx 0.06$. It is well known that ionic strength and the presence of counterions strongly modulate the affinity between macromolecules. Manning (1969) and Seyrek, Dubin, Tribet, \& Gamble (2003) have shown that the binding of polyanions to a positive domain results in a combination of short-range attractive interactions coupled with longer range repulsive interactions due to the presence of charged patches on the protein. Since lys is known to exhibit a multipolar charge distribution (Van de Weert, Andersen, \& Frokjaer, 2004) both interactions are expected in the pectin/lys mixtures. Hence, at low ionic strength, when the Debye length $\kappa^{-1} \approx 0.3 / \sqrt{ } I(\mathrm{~nm})$ is 
large, the effect of salt is mainly to screen the repulsions and thus to strengthen association. At higher ionic strength values, corresponding to a small Debye length, addition of salt weakens the attractions thereby suppressing CP. For the SP/lys systems the maximum in CP occurred around $I=0.03$ (Antonov et al., 2019), which corresponds to a Debye length of $1.7 \mathrm{~nm}$, a value comparable to the size of the lysozyme molecule. The behavior of the BP16.9/lys and BP33.1/lys systems was a little different because the maximum in CP is observed at a higher ionic strength namely $I=0.06$. A possible reason for the small shift of $I_{\max }$ from 0.03 to 0.06 for the BP16.9/1ys and BP33.1/lys systems can be the more pronounced screening of the repulsions due to a stronger aggregation in BP/lys systems as compared to SP/lys systems. This behaviour is thus in correspondence with the formation of much larger complex particles as compared to the particles obtained on the basis of SP (Figure 2).

In addition, similar to the $\mathrm{pH}_{\text {set }}$ values (Fig. 7a) the results presented in Fig. $7 \mathrm{~b}$ and their comparison with those determined for SP/lys systems at corresponding conditions (Antonov et al, 2019) show that the $I_{\text {set }}$ value in BP/lys systems is not dependent on DM, i.e on the overall charge of the pectin molecules, whereas in SP/lys systems the $I_{\text {set }}$ values decrease significantly with the increase of DM. These observations allow to state that the main factor determining the $I_{\text {set }}$ values in SP/lys systems is the overall charge of pectin, whereas in BP/lys systems the $I_{\text {set }}$ values are determined by a combination of the secondary aggregation forces and, to a lesser degree, the overall charge of pectin.

\subsection{Thermodynamic parameters of the binding}

To shed more light on the differences in the complexation behaviour between BP/lys and SP/lys, ITC characterizations were performed for both systems. The raw data from the ITC measurements of the SP16.2/lys system are presented in the inset Figure 8a'. The peak areas correspond to the energies released by the cell containing pectin at each lys injection. The binding isotherm of this system is determined as the integrated area of the heat peaks plotted against the molar ratio of the components (Figure 8a). By determining the thermodynamic parameters of the $\mathrm{CP}$ of proteins or polysacharides with a ligand, it is possible to establish whether $\mathrm{CP}$ is favored by enthalpy or entropy. Because all the injections throughout the analysis released energy, the $\mathrm{CP}$ between lys and SP (Figs. 8a,c,e) and lys and BP (Figs. 8b, d, f) is exothermic. The binding isotherms of all the pectin/lys systems have one inflection point. It is interesting to compare the $\mathrm{CP}$ of the pectin/lys systems with that of pectin/ $\beta$-lactoglobulin systems studied before (Girard et al, 2003a). The binding isotherms of the later system have two inflection points and they reveal 
the formation of soluble intrapolymer complexes (C1) further followed by their aggregation in interpolymer complexes (C2). For that reason, the authors used a "2-site model" interpreted as a "2- step model". In the first step, the amount of $\beta$-lg complexed with pectin decreased with each injection until pectin was saturated. Then, the second step began with a plateau where the same amount of $\beta$-lg molecules interact in each injection. Pectin became gradually saturated with $\beta$-lg until no more energy was released. Girard et al (2003a) assumed that the existence of two steps involved in the pectin/ $\beta$-lactoglobulin $\mathrm{CP}$ process is in agreement with the Tainaka theory (Tainaka, 1979,1980), which states that the complexes are formed in two steps passing from the molecular to the aggregated state. However, the presence of just one inflection point in the binding isotherms in our systems does not mean that the $\mathrm{CP}$ process in the given case is in disagreement with the Tainaka theory. The presence of one inflection point in the binding isotherm is rather a more typical phenomenon for ITC measurements of complex protein-polysaccharide systems (Harnsilawat, Pongsawatmanit, McClements, 2006: Schmitt, da Silva, Bovay, Rami-Shojaei, Frossard, Kolodziejczyk, Leser, 2005; de Souza, Bai, Gonçalves, M. Bastos, 2009). It can be explained by the insignificant concentration range corresponding to the formation of water soluble complexes in these systems (see Fig. 1), or in other words, the absence of a clear isotherm (C1). Moreover, the nonspecific nature of the interactions and the fact that the carboxyl group is the only interacting group on the pectin, would further result in a one-step process.

\section{FIGURE 8}

Curvefitting of the binding isotherms was used to determine the enthalpic $(\Delta \mathrm{H})$ and entropic $(\Delta \mathrm{S})$ contributions of the $\mathrm{CP}$, the binding constant $\mathrm{K}$, and the stoichiometry $\mathrm{n}$ of the complexes at $23^{\circ} \mathrm{C}$

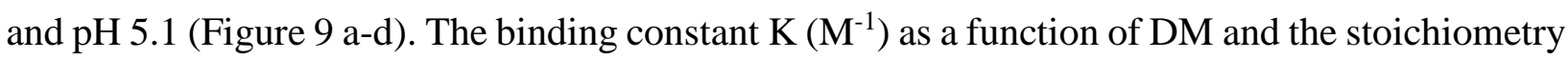
values $\mathrm{n}$ (mole lys/mole pectin) as a function of DM for all systems are presented in Figures 9a and $9 \mathrm{~b}$ respectively. The binding constant of the low methoxylated pectin is more than 50 times higher as compared with that of high methoxylated pectin $\left(11.8 \cdot 10^{-6} \mathrm{M}^{-1}\right.$ and $2.2 \cdot 10^{-7}$ for SP16.2/lys and SP77.7/lys systems respectively). For pectin/ $\beta$-lg systems, the binding constants of the first step were not statistically different, and for the second step this parameter for low methoxylated pectin/ $\beta$-lg was about 8 times higher than for high methoxylated pectin $/ \beta$-lg systems. The effect of DM with pectin/lys systems is thus much more pronounced than that observed for low methoxylated SP/ $\beta$-lg and high methoxylated SP/ $\beta$-lg systems (Girard et al, 2003a). These differences between the different systems suggest that minor differences in the origin of the forces determining $\mathrm{CP}$ in these systems affect the binding constants. Interactions between $\beta$-lg and pectin occurred through ionic and hydrogen bonds, whereas hydrophobic interactions were negligible 
(Girard, Turgeon, Gauthier, 2002). However, in our case hydrophobic interactions may also play an appreciable role together with Coulomb forces. As compared to the effect of DM, the pattern of methylester distribution has a more limited effect on the binding constant. Fig. 9a shows that for all DM values the binding constant for the systems including SP is slightly higher as compared with that of BP. The differences are however very limited, especially when compared to the results of Celus et al. (2018) for binding of pectin with $\mathrm{Zn}^{2+}$, where the binding constants of the $\mathrm{BP}$ at low DM were a factor of 30 higher than those of SP. This is possibly caused by the larger size of lys as compared to $\mathrm{Zn}^{2+}$, which makes the local pattern of ester distribution less relevant for the binding strength. Saturation of the SP16.2 and BP16.9 with lys was reached at lys/pectin molar ratios of about 17:1 and 15:1 mole/mole respectively, which are ratios similar to those determined by turbidimetric titration (16:1 and 15:1). These stoichiometric values decreased monotonically with increasing DM values. Corresponding values for SP66.5 and BP68.1 determined by ITC were $8: 1$ and 4.5:1 respectively.

\section{FIGURE 9}

Despite the moderate effects of the local pattern of charge distribution on the binding constants characterizing the binding of SP and BP with lys, significant differences in the morphology of the formed complexes (Figures 4, 5 and 6) as well as their sensitivity to $\mathrm{pH}$ and $I$ (Figure 7) were observed. Hence, differences in complex formation cannot directly be related to differences in binding strength. First, it should be noted that the formed complexes may contain both lys that underwent actual binding as well as lys that got entrapped in the complexes, whereas the ITC measurements only allow to characterize the first type. Figures $9 \mathrm{c}$ and $9 \mathrm{~d}$ clearly show that under all conditions, the enthalpy contribution dominates over the entropy contribution. The enthalpy and entropy changes remain almost the same when replacing SP16.2 with SP38.2 and BP16.9 with BP33.1, which indicates the absence of significant structural changes in the lys molecule upon complexation (Fig. 9c,d). However, when exchanging SP16.2 with SP77.7, the enthalpy change significantly reduces namely from $-33.5 \mathrm{~kJ} / \mathrm{mol}$ to $10.9 \mathrm{~kJ} / \mathrm{mol}$ or from $8.01 \mathrm{kcal} / \mathrm{mol}$ to -2.6 $\mathrm{kcal} / \mathrm{mol}$. This change is much more pronounced than in the systems with lactoglobulin (which was from $14 \mathrm{kcal} / \mathrm{mol}$ to $10 \mathrm{kcal} / \mathrm{mol}$ ) (Girard et al, 2003). When comparing BP and SP, it becomes clear that mainly at high DM values, where other than electrostatic forces come into play, the relative contributions of entropy and enthalpy to the interaction process are rather different. At high DM, the entropy gain only becomes a significant contribution in the interaction energy for SP. The increasing disorder resulting in the entropy change can result from the release of bound water during complexation. However, as this entropy change significantly increases with DM while the complexation with lys reduces, this can indicate the presence of more pronounced 
contributions of hydrophobic forces, which are generally entropy-driven (Ross and Subramanian 1981). In conclusion, although the ITC measurements reveal differences in the binding behavior of lys with either SP or BP, further research is required to elucidate the precise relations between the molecular level polyelectrolyte binding behaviour and the macroscopic complexation behavior.

\section{Conclusions}

The CP of lys with pectin varying in local charge density has both common and specific features. Both SP and BP form water-soluble complexes in a very small composition range (q between $7 \cdot 10^{-}$ ${ }^{3}$ and $3 \cdot 10^{-2}$ ) depending on DM, and water insoluble complexes at q above $7 \cdot 10^{-3}-0.015$ and less than 1.5-3.8 depending on DM. Optical microscopy shows that pectin with a blockwise distribution of charges forms very large complex particles at all DM values studied, whereas pectin with a statistical distribution of charges forms considerably smaller complex particles, the average size of which is strongly dependent on DM. Separation of the complex pectin/lys systems by centrifugation and isolation of the complex phase showed that pectin with a blockwise charge distribution forms a gel-like complex phase at all DM values studied. Moreover, in contrast to pectin with a statistical charge distribution, pectin with a blockwise charge distribution does not form liquid coacervates at high DM values.

The critical Iset values above which complexes do not form is about the same for both types of pectin (0.10-0.11). According to De Vries (2004) and De Vries et al. (2003) this indicates there is only a small difference in the strength of CP of lys with SP and BP. It is interesting that the critical Iset and pHset values of BP/lys systems, above which complexes did not form, are insensitive to DM, whereas they decrease significantly for SP/lys systems with the increase of DM. The effect of $I$ on the $\mathrm{CP}$ of lys with pectin has a nonmonotonic character displaying a maximum at $I=0.03$ for the SP/lys system and $I=0.06$ for the BP/lys system. This ionic strength corresponds to a Debye length of approximately $1.7 \mathrm{~nm}$, a value comparable to the size of the lys molecule.

The distribution of BP and SP within the complex particles has an irregular character and it is different for high DM values. The binding constant and the stoichiometry ratio of the systems with SP are slightly higher than those of BP at the same DM, and these values decrease sharply (50 times) with the increase of DM. Negative changes in enthalpy of BP/lys system with the increase of DM from 16 till $68 \%$ (change of $\Delta \mathrm{H}$ from $-41 \mathrm{~kJ} / \mathrm{mol}$ to $-58 \mathrm{~kJ} / \mathrm{mol}$ ) give evidence of the formation of a large quantity of hydrogen bonds or Van der Waals interactions during CP. 
537 Blockwise distribution of methoxyl groups enhances pectin/lysozyme complex aggregation.

538 Phase transition compositions depend weakly on methoxyl group distribution.

539 Critical ionic strength of complexation at low DM is independent of methoxyl group distribution.

540 Critical $\mathrm{pH}$ and ionic strength of complexation with blockwise pectin are insensitive to DM.

541 Binding constant is larger with statistical methoxyl group distribution.

542

ACKNOWLEDGMENT

Y.A. Antonov is grateful to KU Leuven for financial support from the Soft Matter Rheology and Technology group.

\section{References}

548 Antonov, Y.A., Lashko, N.P., Glotova, Y.K., Malovikova, A., Markovich, O. (1996). Effect of

549 the structural features of pectins and alginates on their thermodynamic compatibility with gelatin in aqueous media. Food Hydrocolloids, 10, 1-9.

Antonov, Y.A, Zhuravleva, I.L., Cardinaels R.M. \& Moldenaers, P. (2015). Structural studies on the interaction of lysozyme with dextran sulfate. Food Hydrocolloids, 44, 71-80. sodium caseinate and micellar casein in aqueous buffered solutions. Food Hydrocolloids, 62, 102-118.

Antonov, Y. Cardinaels, R., \& Moldenaers P. (2017). Specific effect of the linear charge density of the acid polysaccharide on thermal aggregation/disaggregation processes in complex carrageenan/lysozyme systems. Food Hydrocolloids, 70, 8-13.

Antonov, Y., Zhuravleva, I., Cardinaels, R., \& Moldenaers. P. (2018). Macromolecular complexes of lysozyme with kappa carrageenan. Food Hydrocolloids, 74, 227-238.

Antonov, Y., Celus, M, Kyomugasho, C., Hendrickx, M.,Moldenaers,P., Cardinaels,R. (2019). Complexation of Pectins Varying in Overall Charge with Lysozyme in Aqueous Buffered Solutions., Food Hydrocolloids, in press.

Aune, K.C., \& Tanford C. (1969). Thermodynamics of the denaturation of lysozyme by guanidine hydrochloride. I. Dependence on pH at 25 degrees. Biochemistry. 8(11), 45794585.

Bharti, B., Meissner, J., Klapp, S. H. L. \& Findenegg, G. H. (2014). Bridging Interaction of Protein with Silica Nanoparticles: Influence of pH, Ionic Strength and Protein Concentration, 
Brown, A. (2009) Analysis of Cooperativity by Isothermal Titration Calorimetry. Int. J. Mol. Sci., $10(8), 3457-3477$.

Carlsson, F., Lines, P., \& Malmsten, M. (2001). Monte Carlo simulations of polyelectrolyteprotein complexation. J. Phys. Chem. B, 105, 9040-9049.

Cegielska-Radziejewaka,R., Lesnierowski,G., Kijowski, J. (2008). Properties and application of egg white lysozyme and its modified preparations-A review. Pol. J. Food Nutr.Sci., (1), pp. 510.

Celus, M., Kyomugasho, C., Kermani, Z. J., Roggen, K., Loey, A. M. Van, Grauwet, T., \& Hendrickx, M. E. (2017). Fe2+ adsorption on citrus pectin is influenced by the degree and pattern of methylesterification. Food Hydrocolloids, 73, 101-109.

Celus, M., Lombardo, S., Kyomugasho, C., Thielemans, W., Hendrickx, M.E., (2018).Isothermal titration calorimetry to study the influence of citrus pectin degree and pattern of methylesterification on $\mathrm{Zn}^{2+}$ interaction, Carbohydrate Polym., 197, 460-468.

Cooper, C. L., Dubin, P. L., Kayitmazer, A. B., \& Turksen, S. (2005). Polyelectrolyte-protein complexes. Current Opinion in Colloid and Interface Science, 10, 52-78.

Da Silva, F. L. B., Lund, M., Jönsson, B., \& Akesson, T. (2006). On the complexation of proteins and polyelectrolytes. Journal of Physical Chemistry B, 110(9), 4459-4464.

Daas P.J.H., Meyer - Hansen K., Schols H.A., De Ruiter G.A., Voragen A.G.J., (1999)

Investigation of the non - esterified galacturonic acid distribution in pectins with endopolygalacturonase. Carbohydrate Research, 318, 135-145.

De Kruif, C. G., \& Tuinier, R. (2001). Polysaccharide protein interactions. Food Hydrocolloids, $15,555-563$.

De Kruif, C. G., Weinbreck, F., \& De Vries, R. (2004). Complex coacervation of proteins and anionic polysaccharides. Current Opinion in Colloid and Interface Science, 9, 340-349.

De Souza, H.K., Bai, G., do Pilar Gonçalves, M., \& Bastos, M. (2009). Whey protein isolatechitosan interactions: A calorimetric and spectroscopy study. Thermochim. Acta 495, 108114

De Vries, R. (2004). Monte Carlo simulations of flexible polyanions complexing with whey proteins at their isoelectric point. Journal of Chemical Physics, 120 (7), 3475-3481.

De Vries, R., Weinbreck, F., \& De Kruif, C. G. (2003). Theory of polyelectrolyte adsorption on heterogeneously charged surfaces applied to soluble protein-polyelectrolyte complexes. Journal of Chemical Physics, 118(10), 4549-4659.

Dickinson, E., \& Pawlowsky, K. (1998). Influence of k-carrageenan on the properties of a 
protein-stabilized emulsion. Food Hydrocolloids, 12, 417-423.

Doublier, J. L., Garnier, C., Renard, D., \& Sanchez, C. (2000). Protein-polysaccharide interactions. Curr. Opin. Colloid Interface Sci., 5, 202-214.

Freyer, M. W., Lewis, E. (2008). Isothermal Titration Calorimetry: Experimental Design, Data Analysis, and Probing Macromolecule/Ligand Binding and Kinetic Interactions. In Methods in Cell Biology, Vol. 84, pp 79-113.

Galazkaa, V.B., Smith, D., Ledward, D.A., Dickinson, E. (1999). Complexes of bovine serum Albumin with sulphated polysaccharides: effects of $\mathrm{pH}$, ionic strength and high pressure treatment. Food Chemistry, 64, 303-310.

Girard, M.,Turgeon, S. L., \& Gauthier, S. F. (2002). Interbiopolymer complexing between $\beta$ lactoglobulin and low- and high-methylated pectin measured by potentiometric titration and ultrafiltration. Food Hydrocolloids 16, 585-591.

Girard, M, Turgeon, S.L, \& Gauthier S.F. (2003a).Thermodynamic parameters of betalactoglobulin -pectin complexes assessed by isothermal titration calorimetry. J Agric Food Chem. 16, 51,(15), 4450-5455.

Girard, M, Turgeon, S.L, \& Gauthier S.F. (2003b). Quantification of the Interactions between $\beta$ lactoglobulin and pectin through capillary electrophoresis analysis. J. Agric. Food Chem. 2003b, 51, 6043-6049.

Gurov, A. N. Gurova, N. V. Leontiev, A. L. Tolstoguzov, V.B. (1988). Equilibrium and nonequilibrium complexes between serum albumin and dextran sulfate- I. Complexing conditions and composition of nonequilibrium complexes. Food Hydrocoll. 2, 267-283.

Harnsilawat, T., Pongsawatmanit, R., Mc Clements, D. (2006). Characterization of $\beta$ lactoglobulin-sodium alginate interactions in aqueous solutions: a calorimetry, light scattering, electrophoretic mobility and solubility study. Food Hydrocolloid, 20, 577-585.

Kabanov, V. A. (1994). Basic properties of soluble interpolyelectrolyte complexes applied to bioengineering and cell transformations. In P. L. Dubin (Ed.), Macromolecular complexes in chemistry and biology (pp. 151-174). Berlin: Springer-Verlag.

Kaibara, K., Okazaki, T., Bohidar, H. B., \& Dubin, P. L. (2000). pH-induced coacervation in complexes of bovine serum albumin and cationic polyelectrolytes. Biomacromolecules, 1(1), 100-107.

Kohn, R. and Luknar,O. (1977). Intermolecular calcium ion binding of polyuronates. Coll Czech. Chem. Commun.,42, 731-744.

Kohn R., Markovič O., Machová E., (1983) De-esterification mode of pectin by pectin esterase of Aspergillus foetidus, tomatoes and alfalfa., Collection of Czechoslovak Chemical Communcications, 33, 264-269. 
Kohn R., (1987) Binding of divalent cations to oligomeric fragments of pectin. Carbohydrate Research, 160, 343-353.

Laneuville, S. I.; Paquin, P.; Turgeon, S. L. (2000). Effect of preparation conditions on the characteristics of whey protein-xanthan gum complexes. Food Hydrocoll, 14, 305-314.

Manning, G. S. (1969). Limiting laws and counterion condensation in polyelectrolyte solutions. 1. Colligative properties. J. Chem. Phys., 51, 924-933.

Mattison, K. W.; Brittain, I. J.; Dubin, P. L. (1995). Protein-polyelectrolyte phase boundaries. Biotechnol. Prog, 11, 632-637.

Mattison, K. W., Dubin, P. L., \& Brittain, I. J. (1998). Complex formation between bovine serum albumin and strong polyelectrolytes: effect of polymer charge density. Journal of Physical Chemistry B, 102, 3830-3836.

Ngouémazong, D. E., Tengweh, F.F., Duvetter, T. Fraeye, I., Van Loey, A., Moldenaers, P., Hendrickx, M.E. (2011). Quantifying structural characteristics of partially de-esterified pectins. Food Hydrocolloids, 25, 434-443.

Parmar, A. S., \& Muschol, M. (2009). Hydration and hydrodynamic interactions of lysozyme: effects of chaotropic vs. kosmotropic ions. Biophys. J., 97, 590-598.

Piculell, L., \& Lindman, B. (1992). Association and segregation in aqueous polymer/polymer, polymer/ surfactant, and surfactant/surfactant mixtures: similarities and differences. $A d v$. Colloid Interface Sci., 41, 149-178.

Proctor VA, Cunningham FE. (1988). The chemistry of lysozyme and its use as a food preservative and a pharmaceutical. Crit Rev Food Sci Nutr.26(4):359-95.

Rezwan, K., Meier, L. P., \& Gauckler, L. (2005). Lysozyme and bovine serum albumin adsorption on uncoated silica and $\mathrm{AlOOH}$-coated silica particles: the influence of positively and negatively charged oxide surface coatings. J. Biomaterials, 26, 43514357.

Ross, P.D., Subramanian, S. (1981). Thermodynamics of protein association reactions. Biochemistry, 20, 3096-3102.

Serov, A. N., Antonov, Y. A., Tolstoguzov, V. B. (1985). Isolation of lactic whey proteins in the form of complexes with apple pectin. Nahrung , 1, 19-30.

Seyrek, E., Dubin, P. L., Tribet, C., \& Gamble, E. A. (2003). Ionic strength dependence of protein-polyelectrolyte interactions. Biomacromolecules, 4(2), 273-282.

Schmitt, C., da Silva, T.P., Bovay, C., Rami-Shojaei, S., Frossard, P., Kolodziejczyk, E., Leser, M.E. (2005). Effect of time on the interfacial and foaming properties of betalactoglobulin/acacia gum electrostatic complexes and coacervates at $\mathrm{pH}$ 4.2. Langmuir 21, 7786-7795. 
Sperber, B. L. H. M., Schols, H. A., Stuart, M. A. C., Norde, W. \& Voragen, A. G. J. (2009). Influence of the overall charge and local charge density of pectin on the complex formation between pectin and beta-lactoglobulin. Food Hydrocoll. 23, 3, 765-772.

Stephen, A.M., Phillips, G.O., Williams, P.A. (2006) Food Polysaccharides and Their Applications. Second edition.Taylor \& Francis Group, LLC, USA, 675p.

Ström, A., Schuster, E., MengGoh, S. (2014). Rheological characterization of acid pectin samples in the absence and presence of monovalent ions. Carbohydrate Polymers, 113,(26), 336-343.

Tainaka, K.-I. (1979). Study of complex coacervation in low concentration by virial expansion method. I. Salt free systems. J. Phys. Soc. Jpn. 46, 1899-1906.

Tainaka, K.-I. (1980). Effect of counterions on complex coacervation. Biopolymers 19, 12891298.

Tolstoguzov, V. B. (1997). Protein-polysaccharide interactions. In Damodaran, S., Paraf, A., (Ed), Food proteins and their applications, (pp. 171-198). New York, Marcel Dekker Inc.

Tolstoguzov V. B. (1986). Functional properties of protein-polysaccharide mixtures. In Mitchell, J. R., Ledward, D. A.,( Eds), Functional Properties of Food Macromolecules, (pp 385415) New York, Elsevier Applied Science Publishers.

Tolstoguzov, V.B. (2003). Some thermodynamic considerations in food formulation. Food Hydrocolloids, 17(1), 1-23.

Tsuboi, A., Izumi, T., Hirata, M., Xia J., Dubin, P. L.,\& Kokufuta, E.(1996). Complexation of proteins with a strong polyanion in an aqueous salt-free system. Langmuir, 12, 62956303.

Valstar, A., Brown, W., \& Almgren, M. (1999). Interactions of globular proteins with surfactants studied with fluorescence probe methods. Langmuir, 15, 2635-2643.

van de Weert M, Andersen MB, \& Frokjaer S. (2004). Complex coacervation of lysozyme and heparin: complex characterization and protein stability. Pharmaceutical Research. 21(12),2354-9.

Weinbreck, F., De Vries, R., Schrooyen, P., \& De Kruif, C. G. (2003). Complex coacervation of whey proteins and gum arabic. Biomacromolecules, 4(2), 293-303.

Williams M.A.K., Buffet G.M.C., Foster T.J., Norton I.T., (2001) Simulation of endoPG digest patterns and implications for the determination of pectin fine structure. Carbohydrate research, 334, 243-250.

Voragen, A.G.J., Coenen, G.J., Verhoef, R.P.,Schols, H.A.(2009). Pectin, a versatile polysaccharide present in plant cell walls Structural Chemistry. 20,263-275.

Xia, J., Dubin, P. L., \& Dautzenberg, H. (1993). Light scattering, electrophoresis, and 
turbidimetry studies of bovine serum albumin-poly-(dimethyldiallylammoniumchloride) complex. Langmuir, 9, 2015-2019.

Xu,A.Y., Melton, L.D., Jameson, G.B., Williams, M.A., Mc Gillivray. (2015). Structural mechanism of complex assemblies: characterisation of beta-lactoglobulin and pectin interactions. Soft Matter, 11, 6790-6799.

Xu,A.Y.,Melton,L.D., Ryan,T.M. (2018). Effects of polysaccharide charge pattern on the microstructure of beta-lactoglobulin-pectin complex coacervates, studied by SAXS and SANS. Food hydrocolloids. 77,952 - 963.

\section{Captions for Figures}

722 Figure 1. The turbidity values at $500 \mathrm{~nm}\left(\tau_{500}\right)$ as a function of q for BP16.9/lys (a, a', a"), BP33.2/lys (b, b', b") and BP68.2/lys (c, c', c", c"') mixtures at $I=0.01, \mathrm{pH} 5.1$ and $23^{\circ} \mathrm{C}$. In the

724 insets ( $\left.a^{\prime}, a^{\prime \prime}, b^{\prime}, b^{\prime \prime}, c^{\prime}, c^{\prime \prime}, c^{\prime \prime}\right)$ qonset and qset indicate the transitions between no complex formation and formation of soluble complexes whereas $\mathrm{q}_{\phi}$ and $\mathrm{q}_{\phi}{ }_{\phi}$ indicate the transitions between formation of soluble and insoluble complexes. $\mathrm{C}^{\mathrm{M}}$ tot $=0.04 \mathrm{wt} \%$. As a reference the results for SP16.2/lys and SP38.2/lys systems are added.

Figure 2. (a) The qonset value of BP/lys systems as a function of DM and $\mathrm{q}_{\phi}$ value of BP/lys systems as a function of DM. (b) The qMax value of BP/lys systems as a function of DM, and 1/qmax value of BP/lys systems as a function of DM, $\mathrm{C}^{\mathrm{M}}{ }_{\text {tot }}=0.04 \mathrm{wt} \%, I=0.01, \mathrm{pH} 5.1$ and $23^{\circ} \mathrm{C}$. Error bars indicate the standard deviation and are smaller than the symbol size in Figure 2a.

Figure 3. The intensity size distribution function for BP16.9/lys (a), BP33.1/lys (b) and BP68.2/lys (c) mixtures. The arrow indicates the direction of change of the average size of the particles from $\mathrm{q}=0.05$ to higher q values. $\mathrm{d}$ ) Average radius of complex particles at $\mathrm{q}_{\mathrm{Max}}$ as a function of DM values for SP/lys and BP/lys systems, $\mathrm{C}_{\text {Tot }}^{\mathrm{M}}=0.04 \mathrm{wt} \%, \mathrm{C}_{\text {Pect }}^{\mathrm{o}}=\mathrm{C}_{\text {Lys }}^{\mathrm{o}}=0.04 \mathrm{wt} \%$, $\mathrm{pH} 5.1, I=0.01$ and $23^{\circ} \mathrm{C}$.

Figure 4. Morphological changes in BP68.2/lys systems as a function of the weight ratio q.

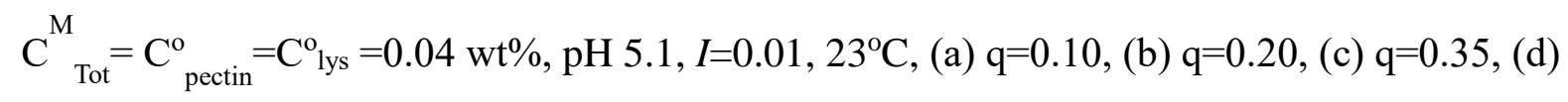
$\mathrm{q}=0.51$. Full length of image is $120 \mathrm{um}$. 
740 Figure 5. Confocal microscopy images of the BP16.9/lys mixtures at different $\mathrm{q}$ values for $\mathrm{C}^{\mathrm{M}}$ tot $741=0.2 \mathrm{wt} \%$. a) $\mathrm{q}=0.05$, b) $\mathrm{q}=0.25$, c) $\mathrm{q}=0.5$, d) $\mathrm{q}=0.9$, e) $\mathrm{q}=1.5, \mathrm{f}) \mathrm{q}=2.0$ for $\mathrm{C}^{\mathrm{M}}$ tot $=0.2 \mathrm{wt} \%$, pH 5.1, $742 I=0.01$ and $23^{\circ} \mathrm{C}$. Full length of images is $410 \mathrm{um}$.

743 Figure 6. Confocal microscopy images of the BP68.2/lys mixtures at different $\mathrm{q}$ values for $\mathrm{C}^{\mathrm{M}}$ tot $744=0.2 \mathrm{wt} \%$, pH 5.1, $I=0.01$ and $23^{\circ} \mathrm{C}$. a) q=0.30, b) q=0.9, c) q=1.5, d) q=2.0. Full length of images 745 is $136 \mathrm{um}$.

746 Figure 7. (a) The turbidity values at $500 \mathrm{~nm}\left(\tau_{500}\right)$ as a function of $\mathrm{pH}$ for BP16.9/lys,

747 BP33.2/lys, and BP68.2/lys systems, $\mathrm{C}_{\text {Tot }}^{\mathrm{M}}=\mathrm{C}_{\text {Pect }}^{\mathrm{o}}=\mathrm{C}_{\text {Lys }}^{\mathrm{o}}=0.04 \mathrm{wt} \%, I=0.01$ and $23^{\circ} \mathrm{C}$. (b) The 748 turbidity values at $500 \mathrm{~nm}\left(\tau_{500}\right)$ as a function of the ionic strength $I$ for BP16.9/lys, BP33.2/lys, 749 and BP68.2/lys systems, $\mathrm{C}_{\text {Tot }}^{\mathrm{M}}=\mathrm{C}_{\text {Pect }}^{\mathrm{o}}=\mathrm{C}_{\text {lys }}^{\mathrm{o}}=0.04 \mathrm{wt} \%, \mathrm{pH} 5.1$ and $23^{\circ} \mathrm{C}$. The inset (b') presents $750 \quad$ Iset as a function of DM.

751

752 Figure 8. (a,c,e) Heat released in each injection versus the ratio between total ligand 753 concentration and total protein concentration for SP16.2/lys (a), SP38.2/lys (c), and SP66.5/lys systems (e). (a') A characteristic titration experiment of the SP16.2/lys system. (b,d,f) Heat released in each injection versus the ratio between total ligand concentration and total protein concentration for BP16.9/lys (b), BP33.2/lys (d), and BP68.2/lys systems (f).

757 Figure 9. (a) The binding constants of the SP/lys and BP/lys systems as a function of DM and 758 (b) stoichiometry values of the SP/lys and BP/lys systems as a function of DM, (c) the enthalpy 759 of binding as a function of DM. The concentrations of lys and highly charged pectin were 1.833 $\mathrm{mM}$ and $0.0128 \mathrm{mM}$ respectively, and the concentration of lys and low charged pectin were $1.3747 \mathrm{mM}$ and $0.0192 \mathrm{mM}$. 


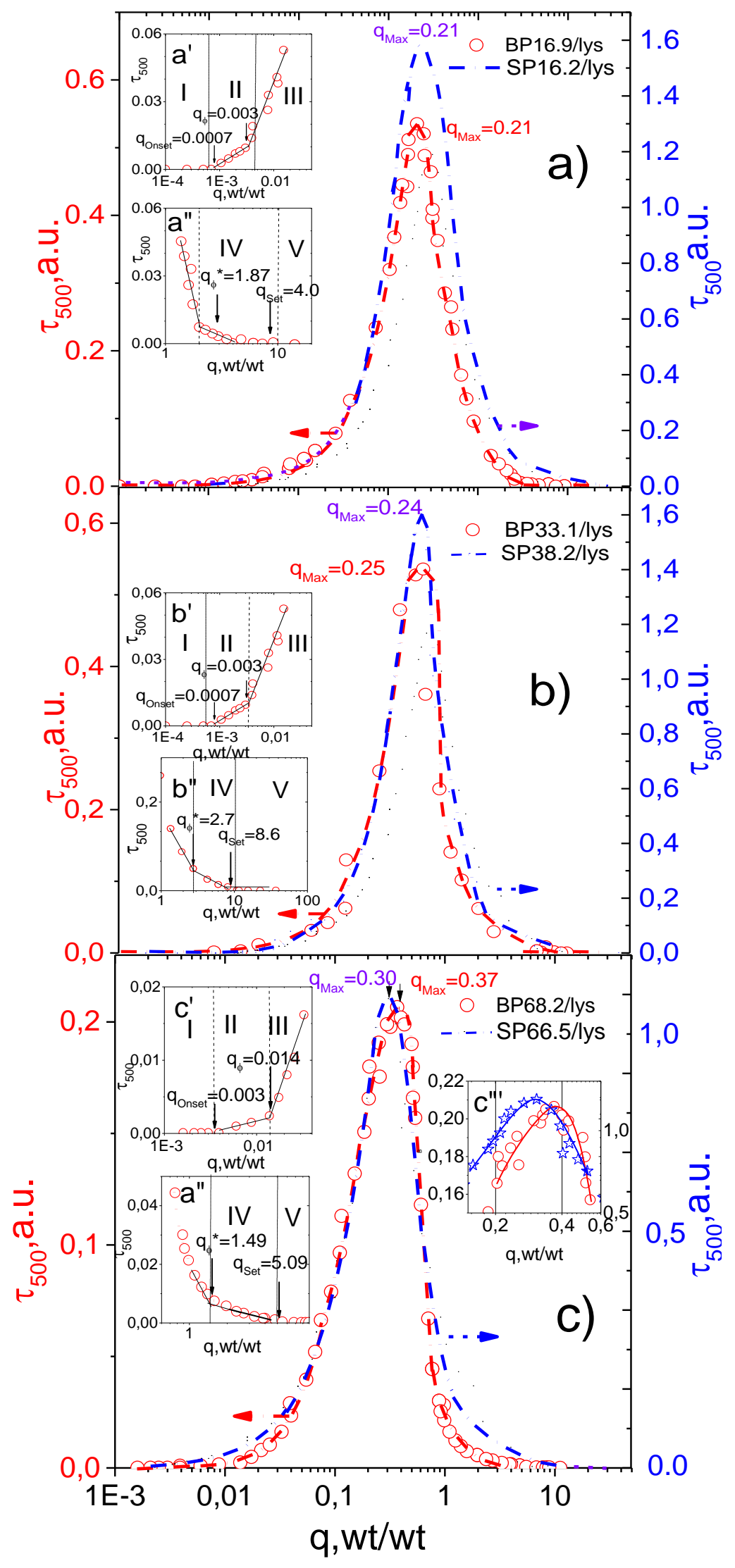

Figure 1

q,wt/wt 

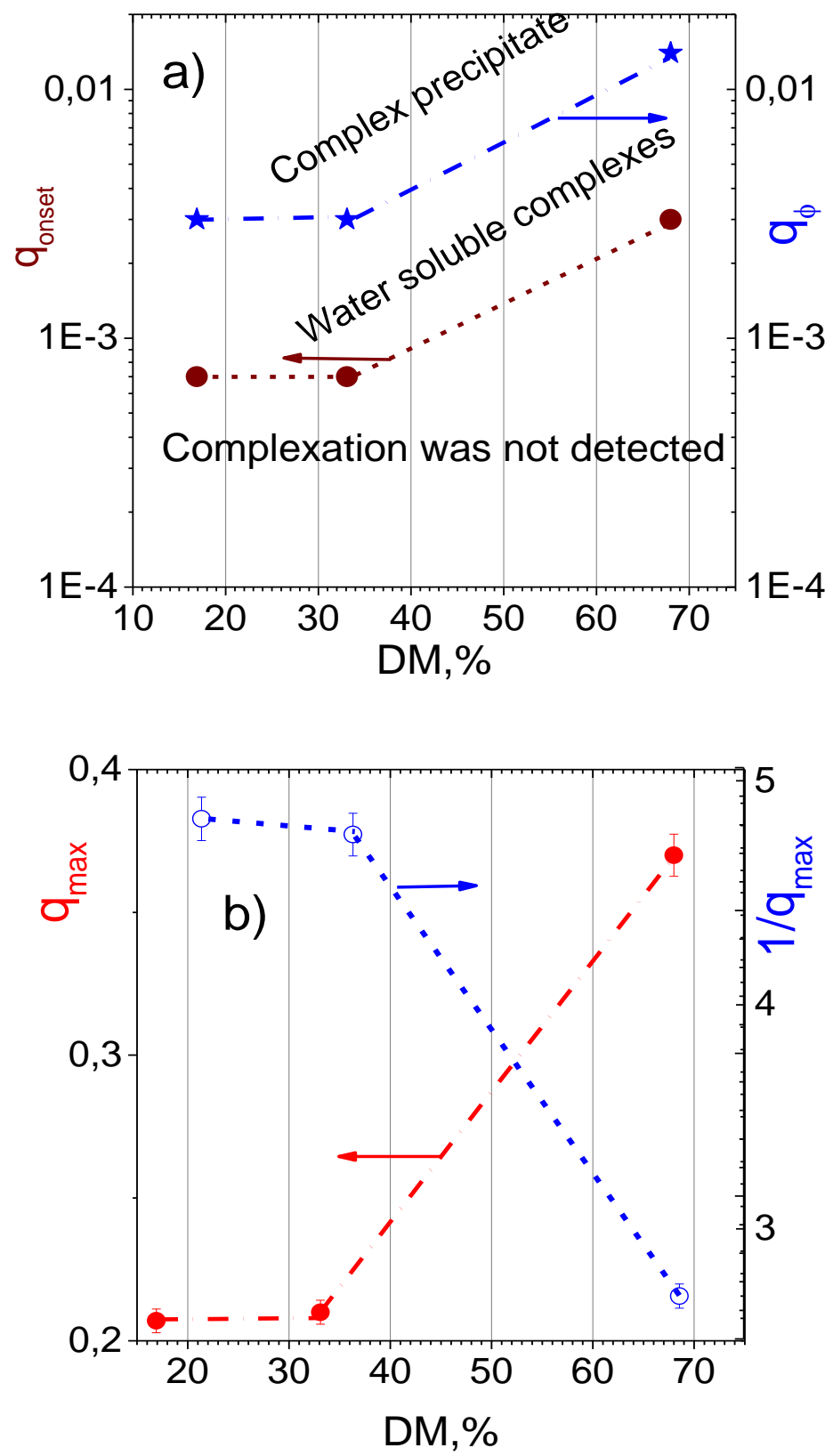

Figure 2 

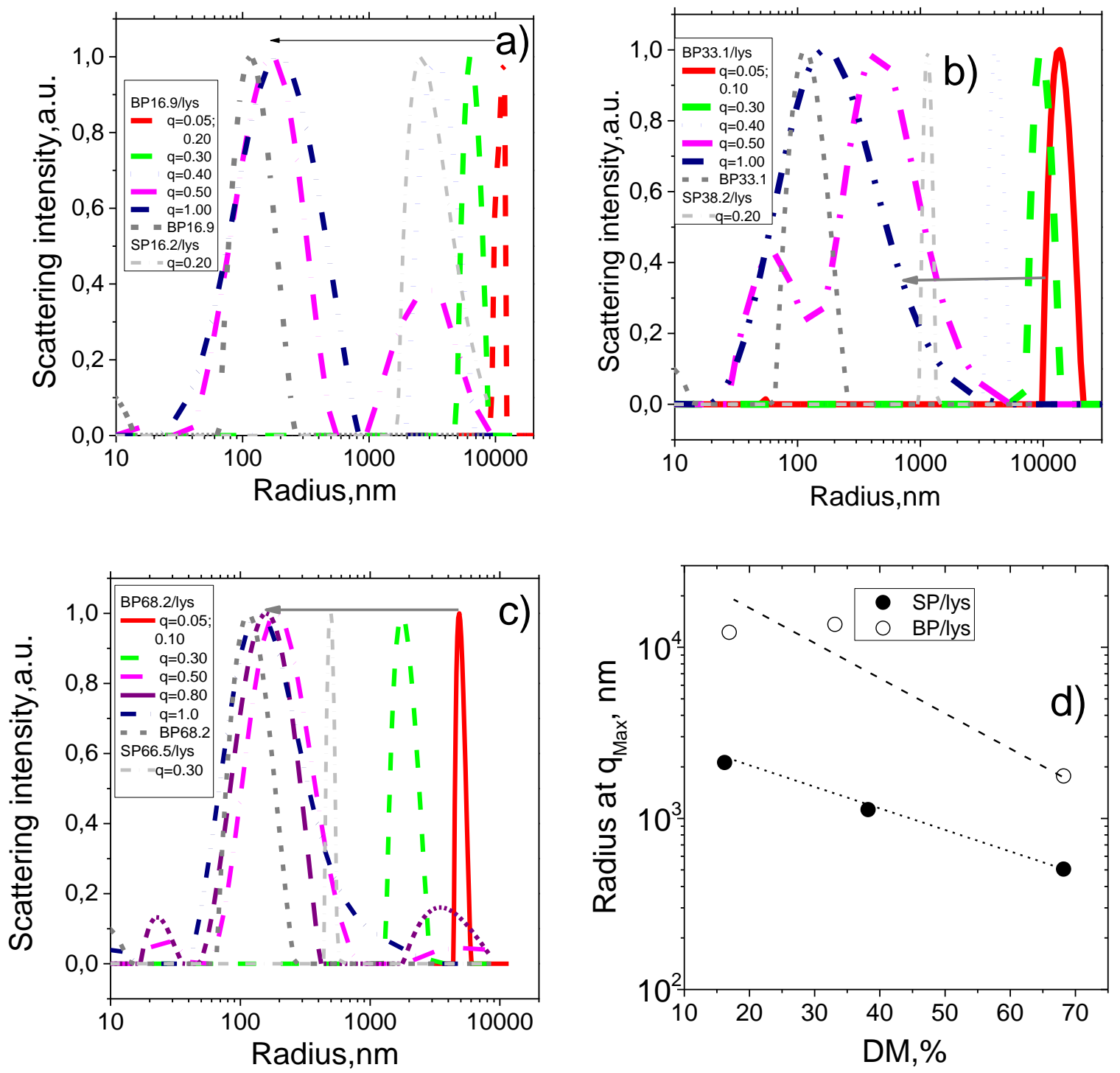

Figure 3 


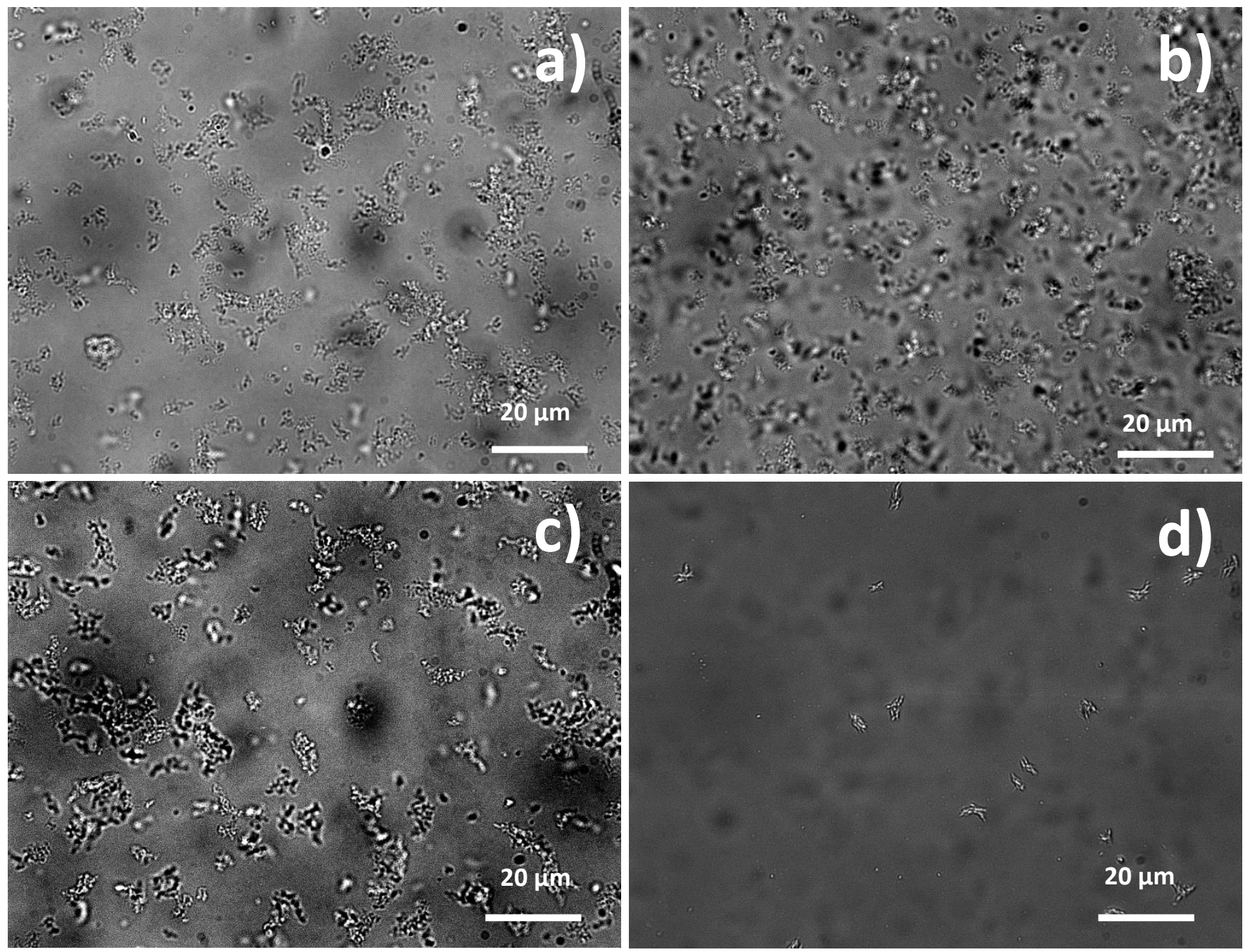

Figure 4 


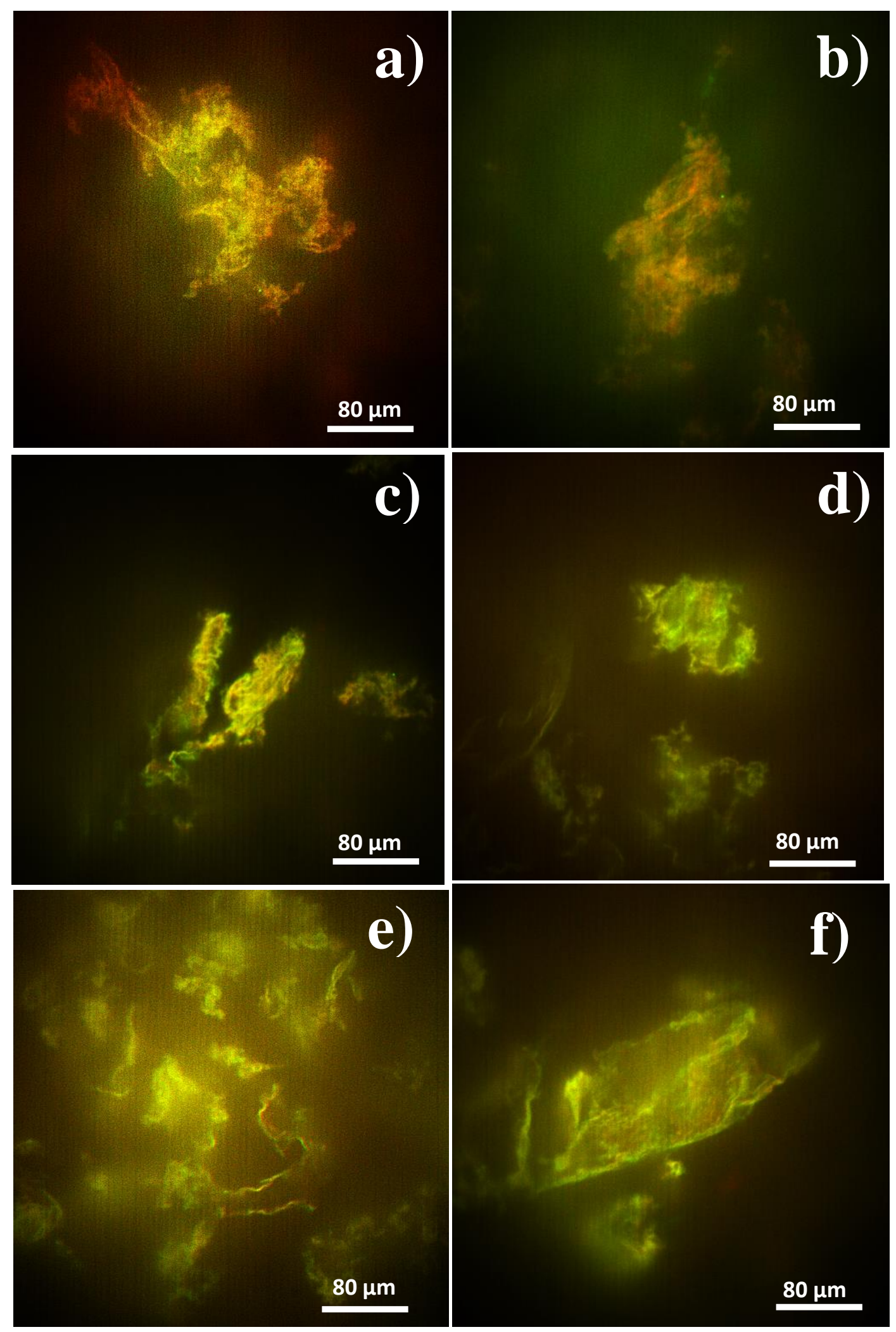

Figure 5 


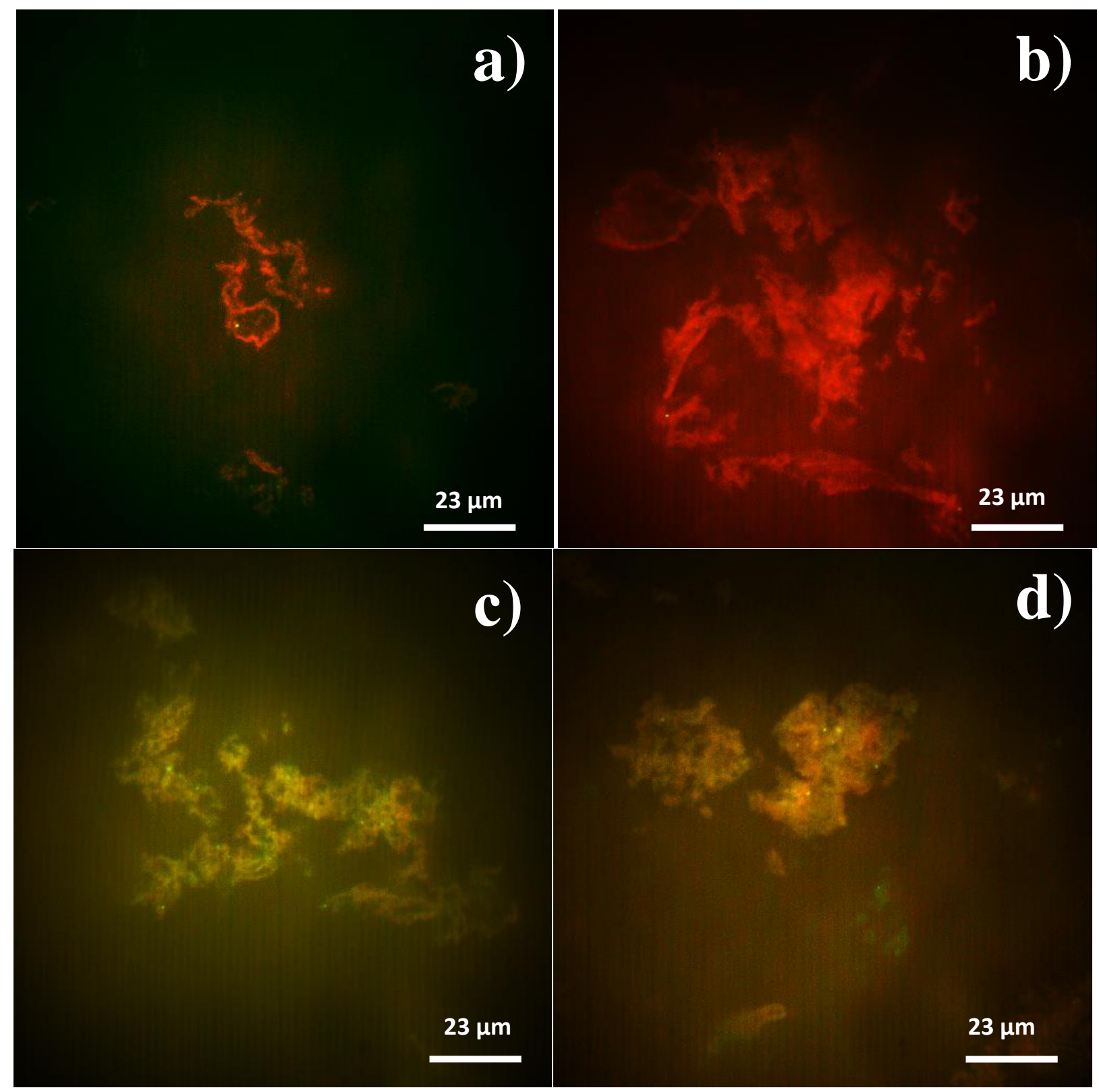

Figure 6 

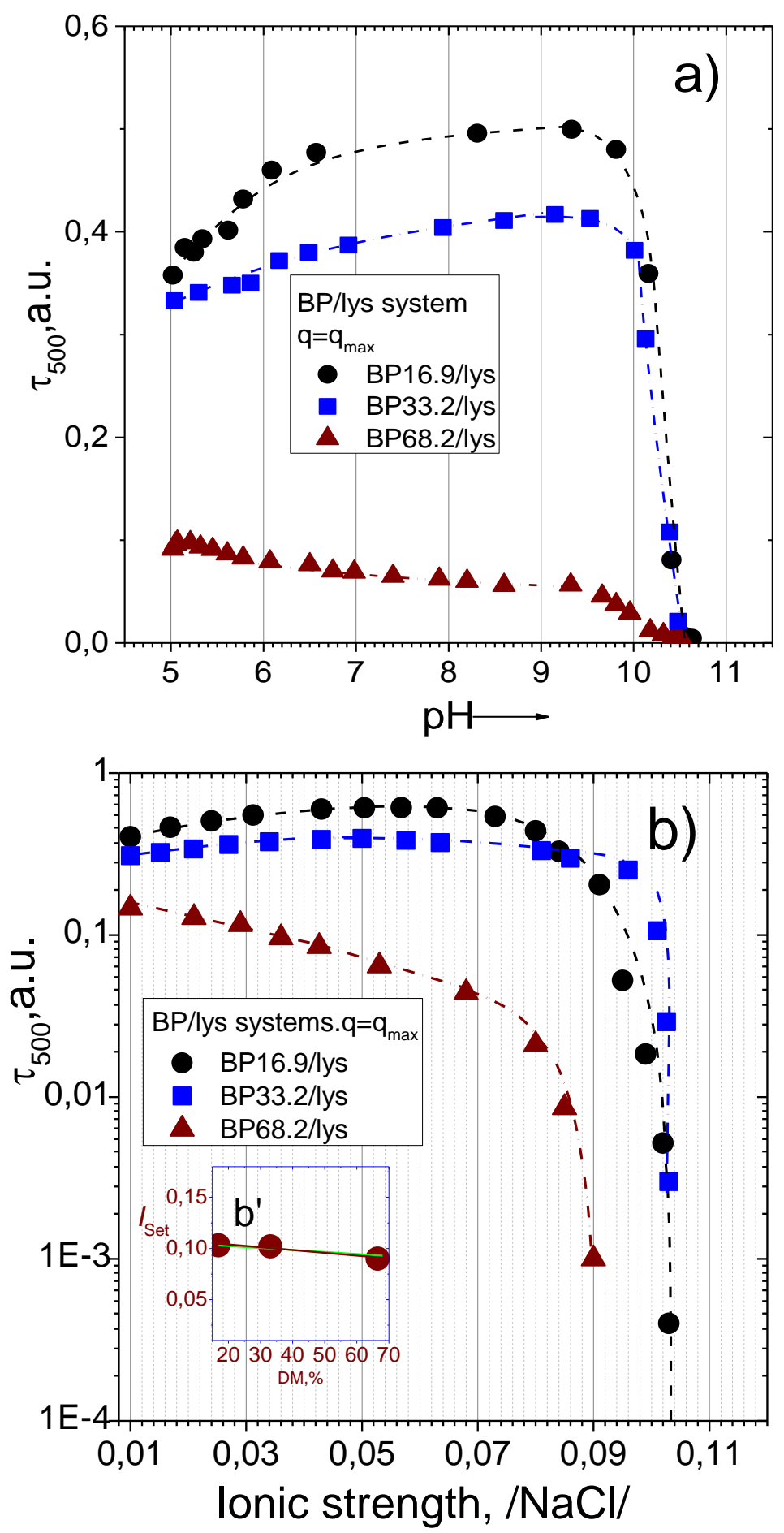

Figure 7 

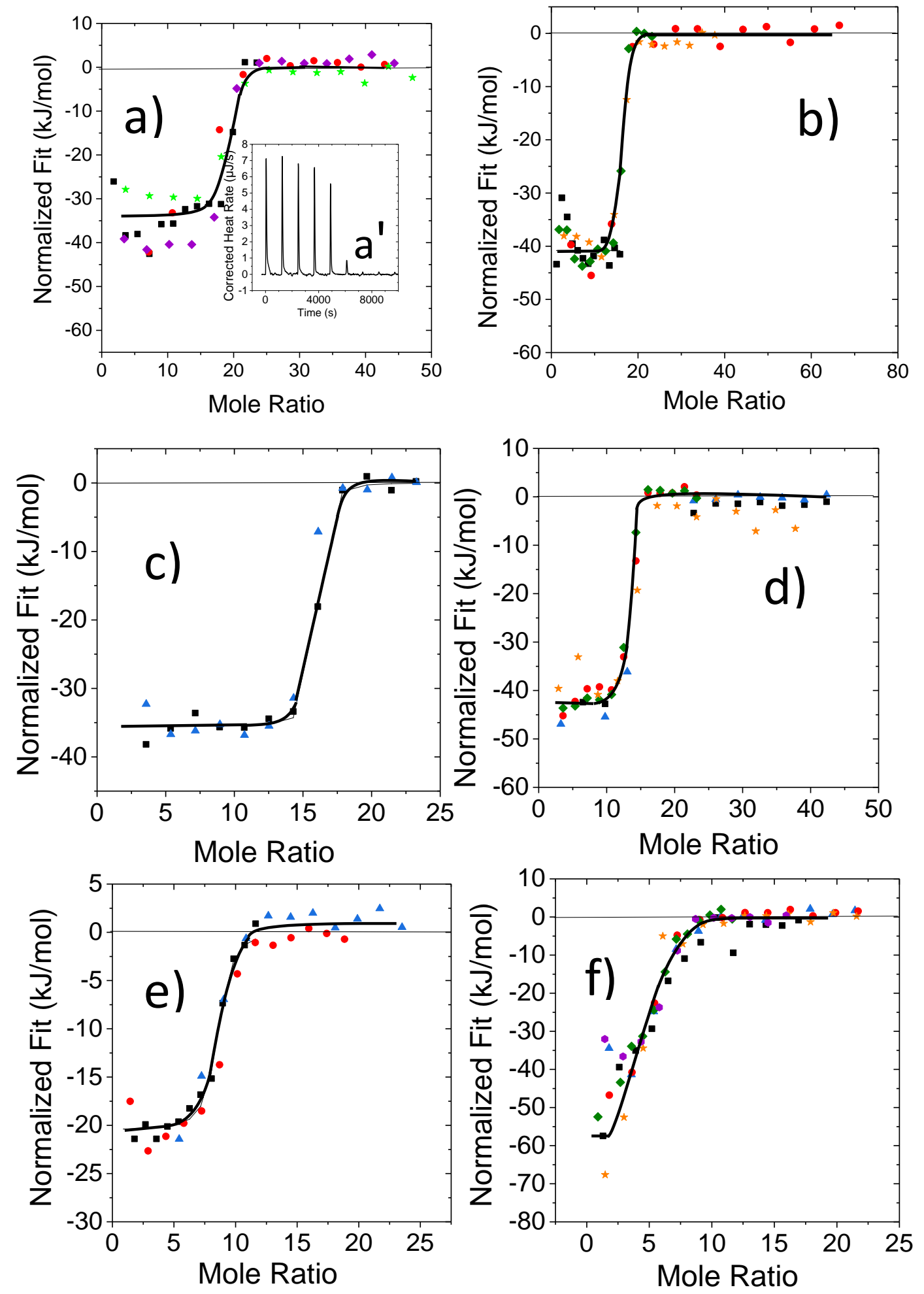

Figure 8 

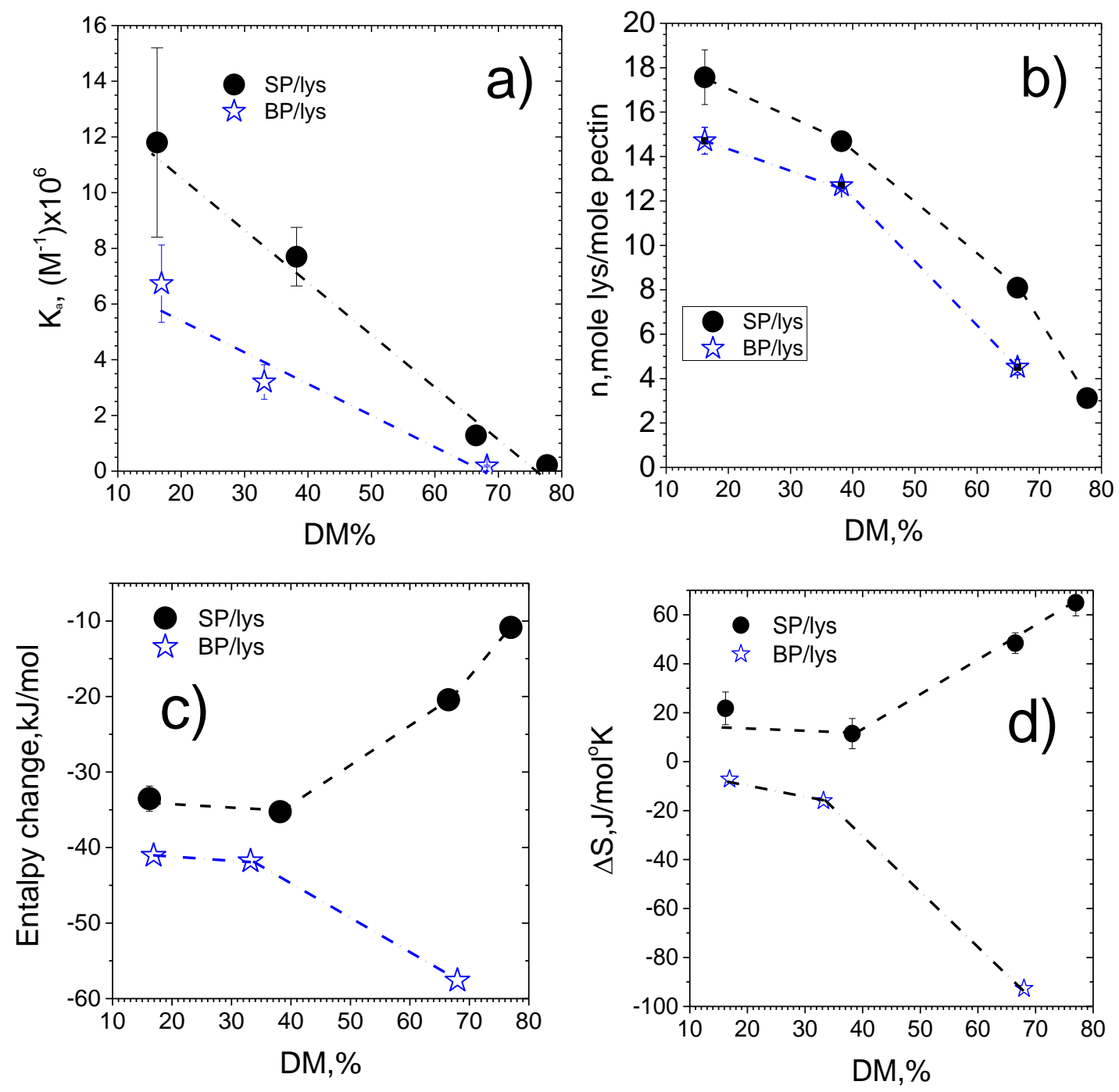

Figure 9 